\title{
Digestive System Adenoma
}

National Cancer Institute

\section{Source}

National Cancer Institute. Digestive System Adenoma. NCI Thesaurus. Code C36207.

A sporadic or less frequently familial neoplasm, arising from the glandular epithelium of the stomach, small intestine, biliary tract, colon, and rectum. It is a polypoid or flat circumscribed lesion. Morphologically, it is characterized by a proliferation of neoplastic glandular cells and it is associated with dysplasia. According to the growth pattern, it may be classified as tubular, villous, or tubulovillous. The dysplasia is classified as mild, moderate, or severe. The frequency of malignant transformation depends on the size of the lesion and the deg ree of dysplasia. Larger adenomas with severe dysplastic changes (sometimes called carcinoma in situ) carry a higher risk of progressing to invasive adenocarcinoma. Gastrointestinal adenomas may present as solitary or multifocal lesions. Familial adenomas tend to be multifocal and carry a higher risk of malignant transformation. 